PITHA 97/34

September 1997

\title{
Signatures of Higgs Bosons in the Top Quark Decay Channel at Hadron Colliders
}

\author{
W. Bernreuther, M. Flesch, and P. Haberl $\mathrm{l}^{\mathrm{m}}$ \\ Institut f. Theoretische Physik, RWTH Aachen, D-52056 Aachen, Germany
}

\begin{abstract}
:
We analyze some signatures of neutral Higgs bosons, produced in high energy proton (anti)proton collisions, which decay primarily to top quarks. This channel is clouded by a large irreducible $t \bar{t}$ background. We investigate the $t \bar{t}$ invariant-mass distribution in the lepton+jets decay mode of $t \bar{t}$ and a $t \bar{t}$ spin-spin correlation in the dilepton decay mode. At the LHC such Higgs bosons can be detected in the $t \bar{t}$ invariant-mass spectrum, and the spin-spin correlation studied by us is also sensitive to a Higgs boson if this particle has a sizeable pseudoscalar component.
\end{abstract}

PACS number(s): 14.65.Ha, 14.80.Bn, 14.80.Cp

\footnotetext{
${ }^{1}$ supported by BMBF contract 057AC9EP.
} 


\section{Introduction}

One of the most important physics issues at future hadron colliders, in particular at the CERN Large Hadron Collider LHC, will be the search for Higgs bosons. While in the standard electroweak theory (SM) one neutral Higgs boson is associated with electroweak symmetry breaking, the situation is more complex in many extensions of the SM. For instance, the well-known (non)supersymmetric two-Higgs doublet extensions of the SM predict the existence of three neutral spin-zero particles: one $\mathrm{CP}$-odd and two CP-even Higgs bosons. The strategies to search for these particles [1] must take into account various possibilities. It may well be that the coupling to weak vector bosons of one of these Higgs particles is suppressed - or even zero, as in the case of a pseudoscalar - whereas its couplings to fermions is enhanced with respect to the SM Higgs couplings.

In this article we investigate the signatures of a heavy Higgs boson in the $t \bar{t}$ decay mode at hadron colliders. Because of the large nonresonant $t \bar{t}$ background this is known to be a very difficult channel for the search of the SM Higgs boson. However, it is important to study this channel in a more general theoretical setting because, as mentioned above, Higgs particles with suppressed couplings to $W$ and $Z$ bosons but enhanced couplings to $t$ quarks may exist. Such a scenario was analyzed in [2], and it was concluded in this paper that under favorable circumstances a scalar or pseudoscalar Higgs boson signal may be observable in the $t \bar{t}$ invariantmass spectrum. Here we extend this analysis in several ways. We consider in the following a neutral Higgs boson $\varphi$ of arbitrary CP nature - i.e., $\varphi$ can be a scalar, pseudoscalar, or a state of undefined CP parity. We investigate in sect. 3 the signal of $\varphi$ in the $t \bar{t}$ invariant-mass spectrum. We consider the case where this spectrum is reconstructed from the single lepton $t \bar{t}$ decay channel which is experimentally rather clean. Moreover, we exploit that in top quark production and decay the top spin is a "good observable" in the sense that due to the extremely short lifetime of the $t$ quark the $t$ spin-polarization and $t \bar{t}$ spin-spin correlations do not get severely diluted by hadronization and can be traced by appropriate correlations among the final state particles into which $t$ and $\bar{t}$ decay [3-8]. The $t$ spin-polarization and $t \bar{t}$ spin-spin correlations are sensitive to the Lorentz structure of the production vertex, and the decay $\varphi \rightarrow t \bar{t}$ leads to characteristic spin correlation signals 99. In sect. 4 we analyze for the double-lepton $t \bar{t}$ decay channel the effects of $\varphi$ production on $t \bar{t}$ spin-spin correlations, and we conclude in sect. 5 .

\section{The $\varphi \rightarrow t \bar{t}$ channel including the QCD back- ground}

In order to be completely general we consider a neutral Higgs boson $\varphi$ with unspecified CP parity. Its coupling to the top quark reads

$$
\mathcal{L}_{Y}=-\left(\sqrt{2} G_{F}\right)^{1 / 2} m_{t}\left(a \bar{t} t+\tilde{a} \bar{t} i \gamma_{5} t\right) \varphi,
$$


where $G_{F}$ is Fermi's constant, $m_{t}$ is the top mass, and $a, \tilde{a}$ are the reduced scalar and pseudoscalar couplings, respectively. (For the SM Higgs boson $a=1, \tilde{a}=0$.) If $a \tilde{a} \neq 0$ then $\varphi$ has undefined CP parity, signaling CP violation in the Higgs sector. This can occur, for instance, in the two-Higgs doublet extension of the SM [10].

In the following we consider a heavy $\varphi$ with mass $m_{\varphi}>2 m_{t}$ with very small couplings to the weak vector bosons, but unsuppressed couplings to $t$ quarks. Such a scenario may be realized in the supersymmetric extension of the SM [2] and also in nonsupersymmetric two-doublet models [1]. Then, for $m_{\varphi}>2 m_{t}$ the mode $\varphi \rightarrow t \bar{t}$ determines the total $\varphi$ decay width. To the extent that the decays of $\varphi$ to $W^{+} W^{-}$ and $Z Z$ are non-negligible the effects discussed in the next section become smaller.

At high energy hadron colliders, for instance at the LHC, $\varphi$ will be produced by gluon-gluon fusion through a virtual top quark loop [11. Scalar and pseudoscalar Higgs production by gluon fusion has been analyzed in detail in the literature [12]. The $\varphi \rightarrow t \bar{t}$ decay channel is affected by the large nonresonant $t \bar{t}$ background. The amplitudes $g g \rightarrow \varphi \rightarrow t \bar{t}$ and $g g \rightarrow t \bar{t}$ interfere and produce at the parton level a characteristic peak-dip structure in the cross section [17, 2, 18] and in other observables [3] if the $t \bar{t}$ invariant mass lies in the vicinity of the Higgs mass. As we are interested, apart from the $t \bar{t}$ invariant mass distribution, also in $t \bar{t}$ spin-spin correlation phenomena we have calculated the $t \bar{t}$ production density matrices for the reactions $q \bar{q} \rightarrow t \bar{t}$ and $g g \rightarrow t \bar{t}$ by taking into account the QCD Born diagrams and the $s$ channel $\varphi$ production diagram. The definitions and results are given in the appendix [19].

The $t \bar{t}$ spin-spin correlations are sensitive to the dynamics of top quark production. This may be exemplified by the the following examples. It is known [22, 4, 5] that close to threshold the gluon-gluon fusion amplitude at Born level yields a $t \bar{t}$ pair in a ${ }^{1} S_{0}$ state. (The QCD Born amplitudes $q \bar{q} \rightarrow t \bar{t}$ leave the $t \bar{t}$ pair in a ${ }^{3} S_{1}$ state.) Consider, on the other hand, $g g \rightarrow \varphi \rightarrow t \bar{t}$. For a scalar and pseudoscalar $\varphi$ the $t \bar{t}$ pair is in a ${ }^{3} P_{0}$ and in a ${ }^{1} S_{0}$ state, respectively. Let us now consider the expectation value of the product $\mathbf{s}_{t} \cdot \mathbf{s}_{\bar{t}}$ of the $t$ and $\bar{t}$ spins. A simple computation yields that $\left\langle\mathbf{s}_{t} \cdot \mathbf{s}_{\bar{t}}\right\rangle=-3 / 4$ if $t \bar{t}$ is produced by gluon fusion at threshold, and $\left\langle\mathbf{s}_{t} \cdot \mathbf{s}_{\bar{t}}\right\rangle=1 / 4(-3 / 4)$ if $t \bar{t}$ is produced by a (pseudo)scalar spin-zero boson and if the $g g \rightarrow t \bar{t}$ background is ignored. In Fig. 1 we have plotted, as a function of the $t \bar{t}$ invariant mass, this expectation value for $g g \rightarrow t \bar{t}$ without and with the inclusion of the $s$ channel scalar, respectively pseudoscalar Higgs boson exchange diagram. The Higgs boson mass $m_{\varphi}=354 \mathrm{GeV}$ was chosen to be close to the $t \bar{t}$ threshold of $350 \mathrm{GeV}$ in order to exhibit the features discussed above. Above the threshold and resonance regions the Higgs effect in $\left\langle\mathbf{s}_{t} \cdot \mathbf{s}_{\bar{t}}\right\rangle$ is actually more pronounced for a pseudoscalar than for a scalar, as can been seen from these figures. This will be further exemplified in sect. 4.

The $t$ and $\bar{t}$ quarks auto-analyze their spins by their parity-violating weak decays. We shall assume that $t \rightarrow W+b$ is the dominant decay mode, as predicted by the SM. It is well-known that the most efficient analyzer of the $t$ spin is the charged lepton from subsequent $W$ decay. Its spin analyzer quality is more than twice as 
high as the $W$ or $b$ quark direction of flight. The corresponding $t$ and $\bar{t}$ decay density matrices are given for instance in [23-25].

In the following section we consider two types of $t \bar{t}$ decay channels: first the "single lepton channel", where the $t$ quark decays semileptonically and the $\bar{t}$ nonleptonically,

$$
t+\bar{t} \rightarrow l^{+} \nu_{l} b+q \bar{q}^{\prime} \bar{b},
$$

and vice versa. These channels have a good signature for top quark identification. Moreover, the $t$ and $\bar{t}$ momenta can be reconstructed up to an ambiguity which can be resolved statistically [26]. These processes are therefore suitable for determining the $t \bar{t}$ invariant-mass spectrum. Second we consider the processes - which we shall call below "double lepton channel" - where both $t$ and $\bar{t}$ decay semileptonically,

$$
t+\bar{t} \rightarrow l^{+} \nu_{l} b+l^{\prime-} \bar{\nu}_{l^{\prime}} \bar{b} .
$$

As mentioned above, correlations among the directions of flight of the charged leptons are best suited to analyze $t \bar{t}$ spin correlations. We will therefore study angular correlations in the double lepton channel.

For obvious reasons we demand the lepton in a semileptonic top decay to be either an electron or a muon. The SM predicts, to good approximation, a fraction of $24 / 81$ of all $t \bar{t}$ events to decay into the single lepton channel and 4/81 into the double lepton channel.

\section{The $t \bar{t}$ invariant mass spectrum}

Throughout our calculation we use the narrow width approximation for $t \bar{t}$ production and decay, which is justified because of $\Gamma_{t} / m_{t}, \Gamma_{W} / m_{W} \ll 1$ and because we are primarily interested in normalized distributions and expectation values of observables. Moreover we take the leptons and the light quarks including the $b$ quark to be massless. Let us start with writing down our "master formula" for the cross section measure for the double lepton channel:

$$
\begin{aligned}
& \int d \sigma\left(p p \rightarrow t \bar{t} X \rightarrow l^{+}+\nu_{l}+b+l^{--}+\bar{\nu}_{l^{\prime}}+\bar{b}+X\right) \\
& =\mathcal{N} \sum_{\lambda=q, \bar{q}, g} \int_{0}^{1} d x_{1} \int_{0}^{1} d x_{2} N_{\lambda}\left(x_{1}\right) N_{\bar{\lambda}}\left(x_{2}\right) \Theta\left(\hat{s}-4 m_{t}^{2}\right) \\
& \times \frac{\alpha_{s}^{2} \beta}{\hat{s}} \int d \Omega_{\hat{\mathbf{k}}} \frac{1}{\eta} \int_{\mu}^{1} d y_{+} y_{+}\left(1-y_{+}\right) \frac{1}{\eta} \int_{\mu}^{1} d y_{-} y_{-}\left(1-y_{-}\right) \\
& \times \int \frac{d \Omega_{\hat{\mathbf{q}}_{+}}}{4 \pi} \int \frac{d \Omega_{\hat{\mathbf{q}}_{-}}}{4 \pi}\left\{A^{(\lambda)}+B^{(\lambda)}\left(\hat{\mathbf{k}} \cdot \hat{\mathbf{q}}_{+}+\hat{\mathbf{k}} \cdot \hat{\mathbf{q}}_{-}\right)\right. \\
& -\left[c_{0}^{(\lambda)} \hat{\mathbf{q}}_{+} \cdot \hat{\mathbf{q}}_{-}+c_{2}^{(\lambda)} \hat{\mathbf{k}} \cdot\left(\hat{\mathbf{q}}_{+} \times \hat{\mathbf{q}}_{-}\right)+c_{4}^{(\lambda)}\left(\hat{\mathbf{p}} \cdot \hat{\mathbf{q}}_{+}\right)\left(\hat{\mathbf{p}} \cdot \hat{\mathbf{q}}_{-}\right)\right. \\
& \left.\left.+c_{5}^{(\lambda)}\left(\hat{\mathbf{k}} \cdot \hat{\mathbf{q}}_{+}\right)\left(\hat{\mathbf{k}} \cdot \hat{\mathbf{q}}_{-}\right)+c_{6}^{(\lambda)}\left(\left(\hat{\mathbf{p}} \cdot \hat{\mathbf{q}}_{+}\right)\left(\hat{\mathbf{k}} \cdot \hat{\mathbf{q}}_{-}\right)+\left(\hat{\mathbf{p}} \cdot \hat{\mathbf{q}}_{-}\right)\left(\hat{\mathbf{k}} \cdot \hat{\mathbf{q}}_{+}\right)\right)\right]\right\}
\end{aligned}
$$




$$
\begin{aligned}
& \times \int_{0}^{\infty} d E_{b} \delta\left(E_{b}-\frac{m_{t}^{2}-m_{W}^{2}}{2 m_{t}}\right) \int \frac{d \Omega_{\hat{\mathbf{q}}_{b}}}{2 \pi} \delta\left(\hat{\mathbf{q}}_{+} \cdot \hat{\mathbf{q}}_{b}-\frac{2 \mu-y_{+}(1+\mu)}{y_{+}(1-\mu)}\right) \\
& \times \int_{0}^{\infty} d E_{\bar{b}} \delta\left(E_{\bar{b}}-\frac{m_{t}^{2}-m_{W}^{2}}{2 m_{t}}\right) \int \frac{d \Omega_{\hat{\mathbf{q}}_{\bar{b}}}}{2 \pi} \delta\left(\hat{\mathbf{q}}_{-} \cdot \hat{\mathbf{q}}_{\bar{b}}-\frac{2 \mu-y_{-}(1+\mu)}{y_{-}(1-\mu)}\right) .
\end{aligned}
$$

In the first line on the r.h.s. $\mathcal{N}=\mathcal{B}\left(t \rightarrow b l \nu_{l}\right)^{2}$ is the square of the semileptonic $t$ branching ratio, and $N_{\lambda}\left(x_{1}\right), N_{\bar{\lambda}}\left(x_{2}\right)$ are the parton distribution functions. The next four lines in eq. (4) represent the differential cross section at the parton level, written in terms of the $t$ momentum direction $\hat{\mathbf{k}}$ in the parton c.m. system, the lepton momentum directions $\hat{\mathbf{q}}_{ \pm}$, and the normalized lepton energies $y_{ \pm}=2 E_{ \pm} / m_{t}$, defined in the $t$ or $\bar{t}$ rest systems, respectively. The minimal value of the normalized lepton energies is $\mu=m_{W}^{2} / m_{t}^{2}$. Forefactors are the strong coupling constant $\alpha_{s}$ and the kinematic factor $\beta=\left(1-4 m_{t}^{2} / \hat{s}\right)^{1 / 2}$ with the parton c.m. energy $\hat{s}=x_{1} x_{2} s$. The factor $\eta=(1-\mu)^{2}(1+2 \mu) / 6$ is chosen such that the lepton energy integrations are normalized to unity.

The matrix element contains the coefficients $A^{(\lambda)}, B^{(\lambda)}, c_{0}^{(\lambda)} \ldots c_{6}^{(\lambda)}$, which depend only on $\hat{s}$ and the cosine of the angle between $\hat{\mathbf{k}}$ and the beam direction, $z=\hat{\mathbf{p}} \cdot \hat{\mathbf{k}}$. They are listed in the appendix. Note that only $A^{(\lambda)}$ contributes to the rate, while the other coefficients lead to $t$ and $\bar{t}$ spin polarization and spin-spin correlations. In particular, the total cross section for the parton subprocess $\lambda \bar{\lambda} \rightarrow t \bar{t}$ is simply

$$
\hat{\sigma}^{(\lambda)}(\hat{s})=\frac{\alpha_{s}^{2} \beta}{\hat{s}} \int d \Omega_{\hat{\mathbf{k}}} A^{(\lambda)}(\hat{s}, z),
$$

which can be computed analytically.

The last two lines in eq. (田) finally give the distribution of $b(\bar{b})$ energies $E_{b}\left(E_{\bar{b}}\right)$ and directions $\hat{\mathbf{q}}_{b}\left(\hat{\mathbf{q}}_{\bar{b}}\right)$, defined again in the $t(\bar{t})$ rest system. The four $\delta$-functions have their origin in the top and $W$ propagators, for which we have used the narrow width approximation. One can see that once the lepton momenta are given, the $b$ $(\bar{b})$ momenta are fixed up to an azimuthal angle around the accompanying lepton, on which the matrix element does not depend.

The cross section for $p \bar{p}$ collisions is simply obtained from eq. (四) by substituting the parton distribution function $N_{\bar{\lambda}}\left(x_{2}\right)$ with $\bar{N}_{\bar{\lambda}}\left(x_{2}\right)$, which enhances the incoherent quark annihilation background. Note that eq. (4) can be as well applied to reactions where one or both of the $t$ quarks decay hadronically; in this case the lepton and neutrino momenta are to be interpreted as the momenta of the (light) quarks into which the $W$ decays, and one has to supplement the appropriate branching ratios.

Let $M_{t \bar{t}}=\sqrt{\left(k_{t}+k_{\bar{t}}\right)^{2}}$ be the invariant mass of the $t \bar{t}$ system. The spectrum $d \sigma / d M_{t \bar{t}}$ is then obtained [2] from multiplying the total parton cross sections $\hat{\sigma}^{(\lambda)}$ at $\hat{s}=M_{t \bar{t}}^{2}$ with the so-called luminosity functions

$$
L^{(\lambda)}\left(M_{t \bar{t}}, s\right)=\frac{2 \tau}{\sqrt{s}} \int_{\tau}^{1 / \tau} \frac{d \zeta}{\zeta} N_{\lambda}(\tau \zeta) N_{\bar{\lambda}}\left(\frac{\tau}{\zeta}\right)
$$

where $\tau=M_{t \bar{t}} / \sqrt{s}$. However, this approach does not account for the experimental difficulty of reconstructing the top momenta, which is present in the single lepton 
channel, and it is not a priori clear to what extent a misidentification might disturb the signal. Apart from that, the implementation of cuts is not straightforward.

We have therefore taken eq. (4) as the starting point for writing a Monte Carlo generator. Importance sampling is performed in the variables $x_{1}$ and $x_{2}$, where we expect the biggest variations (due to the threshold behavior of the parton cross section and the form of the parton distribution functions). As can be seen from eq. (4), the lepton energies can be simulated independently. Moreover, the construction of the $b$ and $\bar{b}$ momenta requires only two (equally distributed) azimuthal angles, as mentioned above.

The obstacle to reconstructing both the $t$ and $\bar{t}$ momenta in the single lepton channel is the missing neutrino momentum. Four-momentum conservation and the various on-shell conditions determine the latter as the solution of a quadratic equation up to a twofold ambiguity. In ref. [26] a strategy was described how to improve on the odds for picking the correct solution: In a first step, cuts are applied on the rapidity of the top quark and the $W$ boson on the hadronic side. Next one tests whether the reconstructed $x_{1}, x_{2}$ lie in the interval $[0,1]$. Finally one chooses that neutrino momentum which - when combined with the lepton momentum - gives a value for the invariant $l-\nu_{l}$ mass which is closest to the physical $W$ mass. This procedure can raise the probability for a correct identification to $86-89 \%$.

It is a simple matter to implement this algorithm into our Monte Carlo generator: Although we know the correct neutrino momentum, we compute for each event the wrong solution and construct a "wrong" top momentum, which we select in 11-14\% of all cases for a determination of $M_{t \bar{t}}$. In addition we apply the following cuts:

$$
|y(t)| \leq 3, \quad p_{T}(l) \geq 20 \mathrm{GeV}
$$

for the rapidity of the top quark and the transverse momenta of the leptons. We will keep these cuts throughout below, also for the double lepton channel in the next section. For the numerics we put $m_{t}=175 \mathrm{GeV}$ and $m_{W}=80.4 \mathrm{GeV}$, and we set the partial widths $\Gamma\left(\varphi \rightarrow W^{+} W^{-}, Z Z\right)$ to zero. We took the parton distribution functions (PDF) from ref. [27], evaluated at the factorization scale $\Lambda=m_{t}$, and convinced ourselves that our results do not change significantly if we vary $\Lambda$ between $m_{t} / 2$ and $2 m_{t}$ or work with other PDF sets [28].

Fig. 2 shows the resulting normalized invariant $t \bar{t}$ mass spectrum $\sigma^{-1} d \sigma / d M_{t \bar{t}}$ for $p \bar{p}$ collisions at $\sqrt{s}=4 \mathrm{TeV}$ in bins of $10 \mathrm{GeV}$, starting at the threshold of $2 m_{t}=350$ $\mathrm{GeV}$. In grey we show the result from the QCD background and superimpose as solid lines the curves which we obtained including the production of a Higgs boson of $m_{\varphi}=400 \mathrm{GeV}$. The respective values of the $\varphi t \bar{t}$ couplings are chosen to be: $2 \mathrm{a}$ ) $a=1, \tilde{a}=0$ (scalar), 2b) $a=0, \tilde{a}=1$ (pseudoscalar), and 2c) $a=\tilde{a}=1$ (undefined $\mathrm{CP}$ parity). Each of the plots in Fig. 2 was produced with 50000 events. This number results from assuming $p \bar{p}$ collisions with $10 \mathrm{fb}^{-1}$ integrated luminosity and a detection efficiency of $1 / 3$ for the single lepton channel.

For the Yukawa couplings used in Fig. 2 the $\varphi$ resonance becomes broader if $\tilde{a} \neq 0$. As a consequence, the contribution to $g g \rightarrow t \bar{t}$ of a $\varphi$ boson with pseudoscalar 
component is, markedly above $\hat{s}>m_{\varphi}^{2}$, more visible than that of a pure scalar. The effect in the normalized spectra shown in Fig. 2 is therefore more pronounced for a $\varphi$ boson with $\tilde{a} \neq 0$ than for a pure scalar $\varphi$. One can see that the typical peak-dip structure in the $M_{t \bar{t}}$ spectrum described in [2] survives at the level of the $t$ and $\bar{t}$ decay products, even if one implements realistic cuts and misidentifies the top quark momentum in $\sim 11 \%$ of all events.

For the upgraded Tevatron an integrated luminosity of $10 \mathrm{fb}^{-1}$ is eventually projected, but at $\sqrt{s}=2 \mathrm{TeV}$. At this c.m. energy no significant signal of a $\varphi$ boson with the Yukawa couplings of Fig. 2 remains above the $t \bar{t}$ background.

The situation clearly improves if we go to $p p$ collisions at LHC energies, one reason being the suppression of the quark annihilation background. In Fig. 3 we show the same series of plots as in Fig. 2, but now for $p p$ at $14 \mathrm{TeV}$. These plots were produced with $1.2 \times 10^{6}$ events. This is a conservative number for the LHC with integrated luminosity of $100 \mathrm{fb}^{-1}$. Qualitatively the signals are comparable to those in Fig. 2, but cleaner due to the larger number of events. The clear signals are of course due to the fact that we chose the Higgs mass $m_{\varphi}=400 \mathrm{GeV}$ to be close to the threshold of $2 m_{t}=350 \mathrm{GeV}$. To study the effect of different Higgs boson masses, we show in Fig. 4 a series of invariant mass distributions obtained for $m_{\varphi}=375$, 400, 450 and $500 \mathrm{GeV}$. In these plots, $\varphi$ was taken to be pseudoscalar with Yukawa couplings $a=0$ and $\tilde{a}=1$.

Let us briefly discuss the statistical significance of our results, for the case of a pseudoscalar with mass $500 \mathrm{GeV}$ (cf. Fig. 4d). Only $84.2 \%$ of the $1.2 \times 10^{6}$ events pass the cuts (7) and are filled into the histogram. If we consider the contents of the five bins below $m_{\varphi}$, i.e. the $M_{t \bar{t}}$ interval between $450 \mathrm{GeV}$ and $500 \mathrm{GeV}$, we find $n_{0}=161765$ events for the background and 164392 events if the $\varphi$ exchange is included, which is a $1.6 \%$ effect. Due to the large number of events this is a significant increase: With a 1 s.d. uncertainty of about $\sqrt{n_{0}} \simeq 402$, this translates into a $6.5 \sigma$ effect. The statistical significance is, of course, higher for the curves depicted in Figs. 4a,b,c.

The Higgs effect decreases if the decays $\varphi \rightarrow W^{+} W^{-}, Z Z$ are non-negligible. If the couplings of $\varphi$ to the weak vector bosons are of SM strength then, using the same number of events, $\varphi$ masses, and Yukawa couplings as in Figs. $4 \mathrm{a}-\mathrm{d}$, the $\varphi$ resonance effect in the $M_{t \bar{t}}$ spectrum becomes insignificant - except possibly for a $\varphi$ with pseudoscalar component and a mass close to $2 m_{t}$.

\section{Spin-spin correlations of the $t \bar{t}$ system}

We have also studied the impact of the $s$-channel $\varphi$-exchange diagram on various correlations among the final state particles. We have concentrated on the double leptonic decay mode and investigated several observables involving the lepton energies and momenta. In particular, the spin-spin correlation $\left\langle\mathbf{s}_{t} \cdot \mathbf{s}_{\bar{t}}\right\rangle$ discussed in sect. 2 
suggests to investigate the observable

$$
\mathcal{O}=\hat{\mathbf{Q}}_{+} \cdot \hat{\mathbf{Q}}_{-},
$$

where $\hat{\mathbf{Q}}_{ \pm}$are the unit vectors of the lepton momenta measured in the hadron c.m. system. In other words, $\mathcal{O}$ is the cosine of the angle between the directions of flight of the two leptons as seen in the laboratory frame. Recall that the lepton momenta $\mathbf{q}_{ \pm}$in $d \sigma$ (4) are defined in the $t$ and $\bar{t}$ rest systems and thus related to $\mathbf{Q}_{ \pm}$by two (rotation-free) boosts.

The grey area in Fig. 5 shows the distribution of $\mathcal{O}$ for $p p$ collisions at $6 \mathrm{TeV}$, as obtained from the background only. One can see that event topologies where the two leptons are back to back or parallel are slightly preferred. In the same figure we show as solid line the resulting distribution if we assume the presence of a pseudoscalar Higgs boson with mass $m_{\varphi}=400 \mathrm{GeV}$ and Yukawa couplings $a=0$, $\tilde{a}=2$. In this case the distribution is shifted towards positive values of $\mathcal{O}$.

In order to quantify this effect one could study the asymmetry between the event numbers with positive and negative $\mathcal{O}$. The same information is of course contained in the expectation value $\langle\mathcal{O}\rangle$. The sensitivity of $\langle\mathcal{O}\rangle$ to the couplings $a$ and $\tilde{a}$ is determined by the 1 s.d. width $\delta \mathcal{O}=\sqrt{\left(\left\langle\mathcal{O}^{2}\right\rangle-\langle\mathcal{O}\rangle^{2}\right) / N}$ where $N$ is the number of events in the sample.

In Fig. 6 we show the theoretical expectation for $\langle\mathcal{O}\rangle$, for $p p$ collisions from 4 to $14 \mathrm{TeV}$. The solid line and the dark grey band represent for a given c.m. energy $\sqrt{s}$ the center value and the $3 \sigma$ interval $[\langle\mathcal{O}\rangle-3 \delta \mathcal{O},\langle\mathcal{O}\rangle+3 \delta \mathcal{O}]$. The width $\delta \mathcal{O}$ was computed with $N_{l l}=2 \times 10^{5}$ events, which is $1 / 6$ of the single lepton events used in the previous section. Also shown in Fig. 6 - as dashed line with light grey band - is the effect on $\langle\mathcal{O}\rangle$ and $\delta \mathcal{O}$ caused by a $m_{\varphi}=400 \mathrm{GeV}$ Higgs boson with pseudoscalar couplings $a=0, \tilde{a}=2$. One can see that this leads to an increase of $\langle\mathcal{O}\rangle$ with a significance above the $3 \sigma$ level. With the same coupling strength and mass, a scalar Higgs boson (i.e. $a=2, \tilde{a}=0$ ) does not yield a significant signal. This is expected from the discussion in sect. 2 .

Both Figs. 5 and 6 were produced with the cuts eq. (可). We observed that the signal gets enhanced by the cut on the lepton transverse momentum.

One might think that the correlation effects increase if one works with the lepton directions $\hat{\mathbf{q}}_{ \pm}$measured in the $t$ and $\bar{t}$ rest systems. Although we consider the double lepton channel, it is possible to reconstruct both the $t$ and $\bar{t}$ momenta [7], albeit with a certain loss in efficiency. We have therefore investigated also the observable

$$
\mathcal{O}^{\prime}=\hat{\mathbf{q}}_{+} \cdot \hat{\mathbf{q}}_{-} \cdot
$$

The results for $\langle\mathcal{O}\rangle$ and $\left\langle\mathcal{O}^{\prime}\right\rangle$ are qualitatively similar. However, in the case of $\left\langle\mathcal{O}^{\prime}\right\rangle$ already the $2 \sigma$ bands overlap, which means that the observable $\mathcal{O}^{\prime}$ is less sensitive to the Yukawa couplings $a$ and $\tilde{a}$ than $\mathcal{O}$.

Finally we mention that neutral Higgs sector $\mathrm{CP}$ violation, i.e. $a \tilde{a} \neq 0$, can be tested with appropriate asymmetries and correlations [3, 21, 25, 29, 30. 


\section{Conclusions}

Extensions of the SM predict a number of neutral Higgs bosons $\varphi$. It is possible that one - or several - of these bosons have very small couplings to $W$ and $Z$ bosons but unsuppressed couplings to top quarks. In this paper we have considered the resonant production of such particles $\varphi$ in the reaction $p+p(\bar{p}) \rightarrow t+\bar{t}+X$. We have studied the effect of $\varphi$ on the $t \bar{t}$ invariant-mass distribution obtained through the single lepton channel. At the upgraded Tevatron $(\sqrt{s}=2 \mathrm{TeV})$ with integrated luminosity of $10 \mathrm{fb}^{-1}$ the signal of $\varphi$ is insignificant - assuming that the Yukawa couplings of $\varphi$ do not exceed SM strength - because of limited statistics and the large $t \bar{t}$ background from quark-antiquark annihilation. For $p \bar{p}$ collisions at $\sqrt{s}=$ $4 \mathrm{TeV}$, assuming again $10 \mathrm{fb}^{-1}$ of integrated luminosity, $\varphi$ bosons become visible in the $t \bar{t}$ invariant-mass spectrum for masses $m_{\varphi}$ around $2 m_{t}$. For $p p$ collisions at LHC energies and luminosities our analysis suggests that $\varphi$ production is detectable for a range of masses $m_{\varphi}$.

If such bosons should be found the analysis of their properties can be supplemented by analyzing $t$ and $\bar{t}$ spin-polarization and spin-spin correlation phenomena in the $\varphi \rightarrow t \bar{t}$ decay channel. The spin-spin correlation that we have studied is, at LHC energies, sensitive to a resonant $\varphi$ contribution if $\varphi$ has a sizeable pseudoscalar coupling to top quarks. This and other correlations and asymmetries should be useful in eventually pinning down the nature of these bosons. Our study was based on the lowest order parton matrix elements. Detailed analyses eventually require the inclusion of QCD radiative corrections, of hadronization and detector effects.

\section{Acknowledgments}

We thank A. Brandenburg for discussions. 


\section{Appendix}

We define a decay density matrix $\rho$ for the process $t \rightarrow \ell^{+} \nu_{\ell} b$ in the narrow width approximation for the $W$ boson by

$$
\frac{\pi}{m_{W} \Gamma_{W}} \delta\left(p_{W}^{2}-m_{W}^{2}\right) \rho_{\alpha^{\prime} \alpha}=\sum_{\ell^{+} \nu_{\ell} b \text { spins }}\left\langle t_{\alpha^{\prime}}\left|\mathcal{T}^{\dagger}\right| \ell^{+} \nu_{\ell} b\right\rangle\left\langle\ell^{+} \nu_{\ell} b|\mathcal{T}| t_{\alpha}\right\rangle
$$

where $p_{W}, m_{W}$ and $\Gamma_{W}$ are the four-momentum, mass and width of the intermediate $W^{+}$, and $\alpha, \alpha^{\prime}$ are the $t$ spin indices. The explicit form of $\rho$, conveniently evaluated in the top quark rest system, can be found e.g. in [25]. The matrix $\bar{\rho}$ for the conjugate decay $\bar{t} \rightarrow \ell^{-} \bar{\nu}_{\ell} \bar{b}$ is obtained from $\rho$ by simple replacements.

Similarly, we define the production density matrices for the partonic process $\lambda \bar{\lambda} \rightarrow t \bar{t}(\lambda=q, g)$ as

$$
R_{\alpha \alpha^{\prime}, \beta \beta^{\prime}}^{(\lambda)}=\frac{1}{g_{s}^{4}} \frac{1}{n_{(\lambda)}} \sum_{\substack{\text { colors } \\ \text { initial spins }}}\left\langle t_{\alpha} \bar{t}_{\beta}|\mathcal{T}| \lambda \bar{\lambda}\right\rangle\left\langle\lambda \bar{\lambda}\left|\mathcal{T}^{\dagger}\right| t_{\alpha^{\prime}} \bar{t}_{\beta^{\prime}}\right\rangle
$$

where the factor $n_{(\lambda)}$ averages over spin and color of the initial state partons: $n_{(q)}=\left(2 N_{C}\right)^{2}=36, n_{(g)}=\left(2\left(N_{C}^{2}-1\right)\right)^{2}=256$. The factor $g_{s}^{4}$ is taken out for convenience. A general discussion of the symmetry properties of these matrices and their decomposition in the $t$ and $\bar{t}$ spin spaces is given in [3]. Here we consider only QCD Born amplitudes and the lowest order $g g \rightarrow \varphi \rightarrow t \bar{t}$ amplitude. Therefore several of the coefficients in the general decomposition vanish, and the matrices $R^{(\lambda)}$ are of the form

$$
\begin{aligned}
R_{\alpha \alpha^{\prime}, \beta \beta^{\prime}}^{(\lambda)}= & A^{(\lambda)} \delta_{\alpha \alpha^{\prime}} \delta_{\beta \beta^{\prime}}+B^{(\lambda)} \hat{k}_{i}\left(\left(\sigma^{i}\right)_{\alpha \alpha^{\prime}} \delta_{\beta \beta^{\prime}}-\delta_{\alpha \alpha^{\prime}}\left(\sigma^{i}\right)_{\beta \beta^{\prime}}\right) \\
& +C_{i j}^{(\lambda)}\left(\sigma^{i}\right)_{\alpha \alpha^{\prime}}\left(\sigma^{j}\right)_{\beta \beta^{\prime}},
\end{aligned}
$$

where $\sigma^{i}$ are the Pauli matrices. In our case, the tensors $C_{i j}^{(\lambda)}$ have the structure

$$
C_{i j}^{(\lambda)}=c_{0}^{(\lambda)} \delta_{i j}+c_{2}^{(\lambda)} \varepsilon_{i j l} \hat{k}_{l}+c_{4}^{(\lambda)} \hat{p}_{i} \hat{p}_{j}+c_{5}^{(\lambda)} \hat{k}_{i} \hat{k}_{j}+c_{6}^{(\lambda)}\left(\hat{p}_{i} \hat{k}_{j}+\hat{p}_{j} \hat{k}_{i}\right)
$$

Here $\hat{\mathbf{k}}$ and $\hat{\mathbf{p}}$ are the unit vectors of the momenta of the top quark and of the initial parton $\lambda$, respectively, defined in the parton c.m. system. (Neglecting transverse parton momenta implies that $\hat{\mathbf{p}}$ is equal to the direction of the proton beam in the laboratory frame.) The squared matrix element of the reaction $\lambda \bar{\lambda} \rightarrow t \bar{t} \rightarrow$ $l^{+} \nu_{l} b+l^{\prime-} \bar{\nu}_{l^{\prime}} \bar{b}$ is then proportional to

$$
\operatorname{Tr}\left[\rho R^{(\lambda)} \bar{\rho}\right] \equiv \rho_{\alpha^{\prime} \alpha} R_{\alpha \alpha^{\prime}, \beta \beta^{\prime}}^{(\lambda)} \bar{\rho}_{\beta^{\prime} \beta},
$$

which leads - after integration over the phase space and folding with parton distribution functions - to the representation of the differential cross section $d \sigma$ given in eq. (4). 
The matrix $R^{(q)}$ gets only contributions from the QCD background, leading to the coefficients

$$
\begin{aligned}
A^{(q)} & =N_{q}\left[1-\frac{\beta^{2}}{2}\left(1-z^{2}\right)\right], & c_{5}^{(q)} & =N_{q}\left[\beta^{2}+z^{2}(1-x)^{2}\right], \\
c_{0}^{(q)} & =N_{q}\left[-\frac{\beta^{2}}{2}\left(1-z^{2}\right)\right], & c_{6}^{(q)} & =N_{q}[-z(1-x)], \\
c_{4}^{(q)} & =N_{q}, & B^{(q)} & =c_{2}^{(q)}=0,
\end{aligned}
$$

with

$$
x=\frac{2 m_{t}}{\sqrt{\hat{s}}}, \quad \beta=\sqrt{1-x^{2}}, \quad z=\hat{\mathbf{p}} \cdot \hat{\mathbf{k}}
$$

and the normalization factor

$$
N_{q}=\frac{1}{4} \frac{C_{F}}{C_{A}}
$$

As usual, the $S U\left(N_{C}\right)$ color factors are $C_{F}=\left(N_{C}^{2}-1\right) /\left(2 N_{C}\right), C_{A}=N_{C}$.

We split the coefficients of the gluon fusion density matrix $R^{(g)}$ into contributions from the QCD background and contributions involving the $\varphi$ exchange diagram:

$$
A^{(g)}=A_{\mathrm{Born}}^{(g)}+A_{\varphi}^{(g)},
$$

and similar for the other coefficients. For the background part we obtain

$$
\begin{aligned}
A_{\text {Born }}^{(g)} & =N_{g}^{(0)}\left[1+x^{2}-\frac{1-\beta^{2} z^{2}}{2}-\frac{x^{4}}{1-\beta^{2} z^{2}}\right] \\
c_{0, \text { Born }}^{(g)} & =N_{g}^{(0)}\left[x^{2}-\frac{1-\beta^{2} z^{2}}{2}-\frac{x^{4}}{1-\beta^{2} z^{2}}\right] \\
c_{4, \text { Born }}^{(g)} & =N_{g}^{(0)}\left[\frac{\beta^{2}\left(1-z^{2}\right)}{1-\beta^{2} z^{2}}\right] \\
c_{5, \text { Born }}^{(g)} & =N_{g}^{(0)} \frac{1-x}{1+x}\left[1+2 x(1+x)+\beta^{2} z^{2}-\frac{x^{2}\left(3+4 x+2 x^{2}\right)}{1-\beta^{2} z^{2}}\right] \\
c_{6, \text { Born }}^{(g)} & =N_{g}^{(0)}[-z(1-x)]\left[\frac{\beta^{2}\left(1-z^{2}\right)}{1-\beta^{2} z^{2}}\right] \\
B_{\text {Born }}^{(g)} & =c_{2, \text { Born }}^{(g)}=0 .
\end{aligned}
$$

For the contributions from $\varphi$ exchange it is convenient to define the following two functions,

$$
\begin{aligned}
D & =\left(-2+\beta^{2} \hat{s} C_{0}\right) \frac{\hat{s}}{\hat{s}-m_{\varphi}^{2}+i m_{\varphi} \Gamma_{\varphi}}, \\
\tilde{D} & =\hat{s} C_{0} \frac{\hat{s}}{\hat{s}-m_{\varphi}^{2}+i m_{\varphi} \Gamma_{\varphi}}
\end{aligned}
$$


which contain the loop integral

$$
C_{0} \equiv C_{0}\left(p_{1}, p_{2}, m_{t}, m_{t}, m_{t}\right)=\frac{1}{2 \hat{s}}\left[\ln \left(\frac{1+\beta}{1-\beta}\right)-i \pi\right]^{2}
$$

as well as the Breit-Wigner form of the $\varphi$ propagator. With the help of $D$ and $\tilde{D}$ the remaining contributions can be written in a compact way:

$$
\begin{aligned}
A_{\varphi}^{(g)} & =N_{g}^{(2)}\left[\beta^{2} a^{2} \operatorname{Re}(D)+\tilde{a}^{2} \operatorname{Re}(\tilde{D})\right] \\
& +N_{g}^{(4)}\left(\beta^{2} a^{2}+\tilde{a}^{2}\right)\left(a^{2}|D|^{2}+\tilde{a}^{2}|\tilde{D}|^{2}\right) \\
B_{\varphi}^{(g)} & =N_{g}^{(2)}(\beta a \tilde{a}) \operatorname{Im}(D-\tilde{D}) \\
c_{0, \varphi}^{(g)} & =N_{g}^{(2)}\left[\beta^{2} a^{2} \operatorname{Re}(D)-\tilde{a}^{2} \operatorname{Re}(\tilde{D})\right] \\
& +N_{g}^{(4)}\left(\beta^{2} a^{2}-\tilde{a}^{2}\right)\left(a^{2}|D|^{2}+\tilde{a}^{2}|\tilde{D}|^{2}\right) \\
c_{2, \varphi}^{(g)} & =N_{g}^{(2)}(-\beta a \tilde{a}) \operatorname{Re}(D+\tilde{D})+N_{g}^{(4)}(-2 \beta a \tilde{a})\left(a^{2}|D|^{2}+\tilde{a}^{2}|\tilde{D}|^{2}\right) \\
c_{5, \varphi}^{(g)} & =N_{g}^{(2)}\left(-2 \beta^{2} a^{2}\right) \operatorname{Re}(D)+N_{g}^{(4)}\left(-2 \beta^{2} a^{2}\right)\left(a^{2}|D|^{2}+\tilde{a}^{2}|\tilde{D}|^{2}\right) \\
c_{4, \varphi}^{(g)} & =c_{6, \varphi}^{(g)}=0 .
\end{aligned}
$$

Here $a$ and $\tilde{a}$ are the reduced scalar and pseudoscalar Yukawa couplings defined in eq. (11). The normalization factors are

$$
\begin{aligned}
N_{g}^{(0)} & =\frac{1}{2}\left(\frac{1}{C_{A}} \frac{1}{1-\beta^{2} z^{2}}-\frac{1}{4 C_{F}}\right) \\
N_{g}^{(2)} & =\frac{1}{2} \frac{1}{C_{F} C_{A}} \frac{1}{32 \pi^{2}}\left(\frac{m_{t}}{v}\right)^{2} \frac{x^{2}}{1-\beta^{2} z^{2}}, \\
N_{g}^{(4)} & =\frac{1}{2} \frac{1}{C_{F}}\left(\frac{1}{32 \pi^{2}}\right)^{2}\left(\frac{m_{t}}{v}\right)^{4} x^{2},
\end{aligned}
$$

with $v=\left(\sqrt{2} G_{F}\right)^{-1 / 2}=246 \mathrm{GeV}$. The coefficients given in this appendix agree with those obtained in [3]. The cross sections for the partonic reactions are determined by $A^{(\lambda)}$, and our expression for $d \hat{\sigma}(g g \rightarrow t \bar{t}) / d z$ agrees with [2]. 


\section{References}

[1] For reviews, see for instance J. Gunion, H. Haber, G. Kane and S. Dawson, The Higgs Hunter's Guide (Addison-Wesley, New York, 1990);

Z. Kunszt, hep-ph/9704263 (1997).

[2] D. Dicus, A. Stange, and S. Willenbrock, Phys. Lett. B 333, 126 (1994).

[3] W. Bernreuther and A. Brandenburg, Phys. Lett. B 314, 104 (1993); Phys. Rev. D 49, 4481 (1994).

[4] T. Arens and L. M. Sehgal, Phys. Lett. B 302, 501 (1993).

[5] A. Brandenburg, Phys. Lett. B 388, 626 (1996).

[6] G. Mahlon and S. Parke, Phys. Rev. D 53, 4886 (1996).

[7] D. Chang, S. Lee, and A. Soumarokov, Phys. Rev. Lett. 77, 1218 (1996).

[8] T. Stelzer and S. Willenbrock, Phys. Lett. B 374, 169 (1996).

[9] See, for instance, W. Bernreuther, A. Brandenburg, and M. Flesch, Phys. Rev. D 56, 90 (1997), and references cited therein.

[10] S. Weinberg, Phys. Rev. D 42, 860 (1990).

[11] H. M. Georgi, S. I. Glashow, M. E. Machacek, and D. V. Nanopoulos, Phys. Rev. Lett. 40, 692 (1978).

[12] In particular the QCD corrections to the lowest order production rates were computed in [13-15] and it was found that they increase the lowest order rates significantly (see also [16]).

[13] A. Djouadi, M. Spira, and P. Zerwas, Phys. Lett. B 264, 440 (1991); D. Graudenz, M. Spira, and P. Zerwas, Phys. Rev. Lett. 70, 1372 (1993); S. Dawson, Nucl. Phys. B359, 283 (1991).

[14] M. Spira, A. Djouadi, D. Graudenz, and P. Zerwas, Nucl. Phys. B453, 17 (1995).

[15] M. Spira, A. Djouadi, D. Graudenz, and P. Zerwas, Phys. Lett. B 318, 347 (1993); S. Dawson, A. Djouadi, and M. Spira, Phys. Rev. Lett. 77, 16 (1996).

[16] Z. Kunszt, S. Moretti, and W. J. Stirling, Z. Phys. C 74, 479 (1997).

[17] K. Gaemers and F. Hoogeveen, Phys. Lett. B 146, 347 (1984).

[18] W. Beenakker et al., Nucl. Phys. B411, 343 (1994).

[19] The remaining nonresonant Yukawa corrections are of no concern to us here; they have been shown to be small in general. See ref. [20] for CP-conserving and [21, 3] for CP-violating Higgs boson exchange in two-Higgs doublet models. The one-loop electroweak corrections to $g g \rightarrow t \bar{t}$ within the SM were computed in 18 and the resulting correction to the $p p \rightarrow t \bar{t} X$ cross section was found to be of the order of a few percent. 
[20] H. Y. Zhou, C. S. Li, and Y. P. Kuang, Phys. Rev. D 55, 4412 (1997).

[21] C.R. Schmidt, M.E. Peskin, Phys. Rev. Lett. 69, 410 (1992).

[22] Y. Hara, Prog. Theor. Phys. 86, 779 (1991).

[23] W. Bernreuther, O. Nachtmann, P. Overmann, and T. Schröder, Nucl. Phys. B388, 53 (1992); B406, 516 (E) (1993).

[24] J. P. Ma and A. Brandenburg, Z. Phys. C 56, 97 (1992).

[25] P. Haberl, O. Nachtmann, and A. Wilch, Phys. Rev. D 53, 4875 (1996).

[26] G. A. Ladinsky, Phys. Rev. D 46, 3789 (1992), 47, 3086(E) (1993).

[27] M. Glück, E. Reya, and A. Vogt, Z. Phys. C 67, 433 (1995).

[28] A. D. Martin, W. J. Stirling, and R. G. Roberts, Phys. Lett. B 354, 155 (1995);

H. L. Lai et al. (CTEQ coll.), Phys. Rev. D 51, 4763 (1995).

[29] A. Brandenburg and J. P. Ma, Phys. Lett. B 298, 211 (1993).

[30] B. Grzadkowski, B. Lampe, and K. J. Abraham, hep-ph/9706489 (1997). 
Figure 1

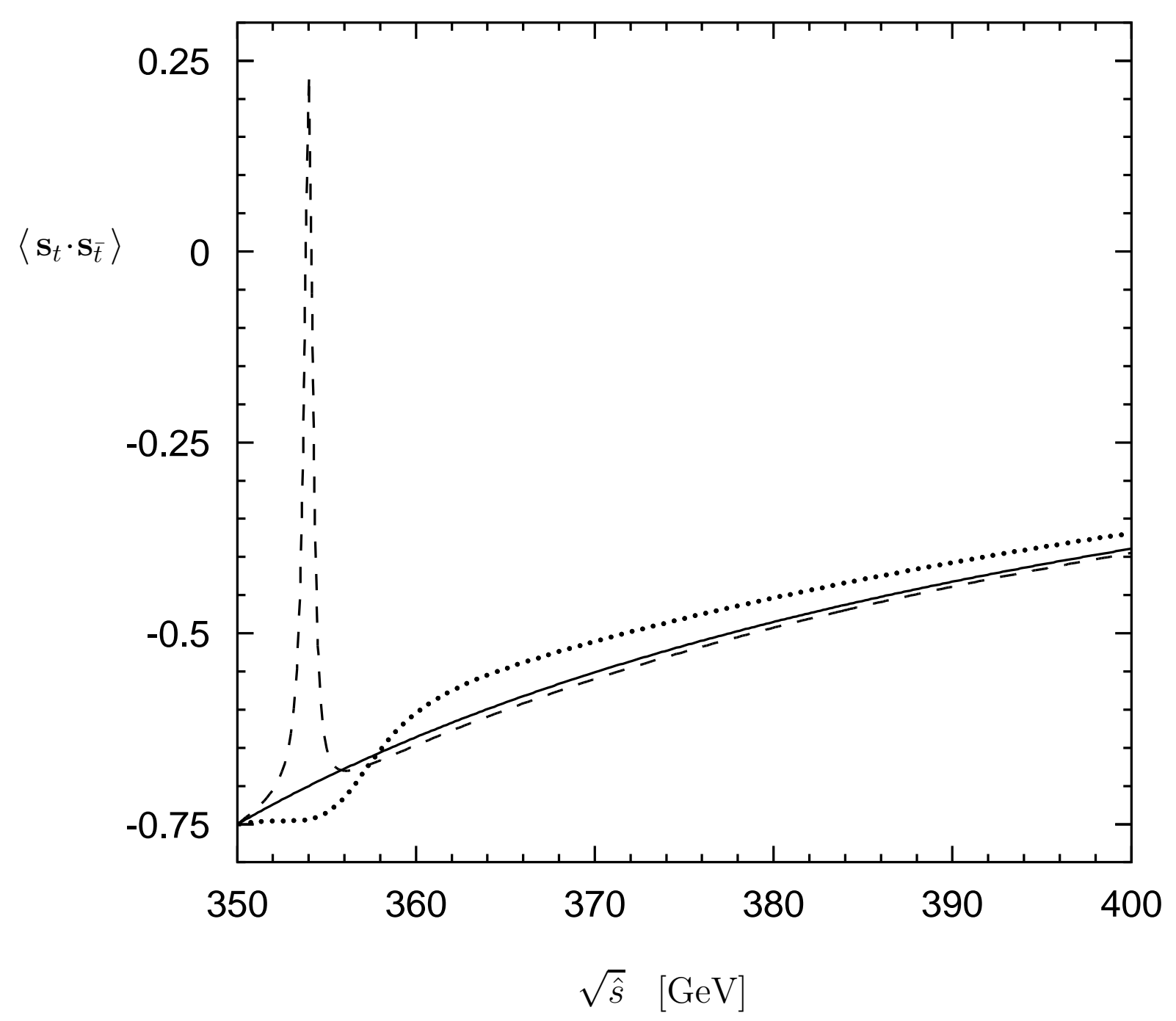

Fig. 1: Expectation value $\left\langle\mathbf{s}_{t} \cdot \mathbf{s}_{\bar{t}}\right\rangle$ of the product of $t$ and $\bar{t}$ spins in the process $g g \rightarrow t \bar{t}$, plotted as function of the parton c.m. energy $\sqrt{\hat{s}}$. The solid line is the result for the background, while the dashed (dotted) line shows the effect of a scalar (pseudoscalar) Higgs boson with mass $m_{\varphi}=354 \mathrm{GeV}$ and reduced Yukawa couplings $a=1, \tilde{a}=0(a=0, \tilde{a}=1)$. 
Figure 2
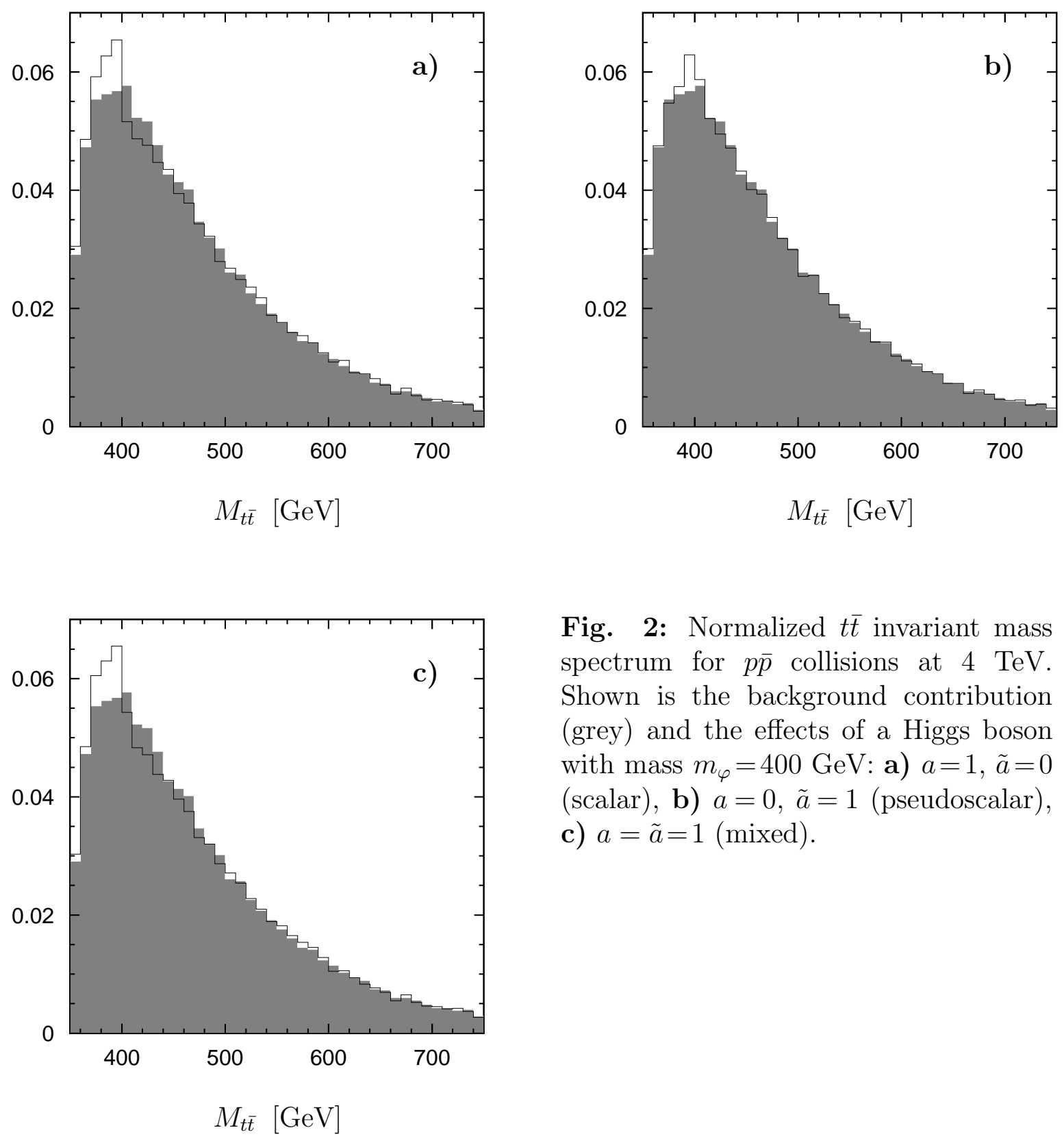

Fig. 2: Normalized $t \bar{t}$ invariant mass spectrum for $p \bar{p}$ collisions at $4 \mathrm{TeV}$. Shown is the background contribution (grey) and the effects of a Higgs boson with mass $m_{\varphi}=400 \mathrm{GeV}$ : a) $a=1, \tilde{a}=0$ (scalar), b) $a=0, \tilde{a}=1$ (pseudoscalar), c) $a=\tilde{a}=1$ (mixed). 
Figure 3
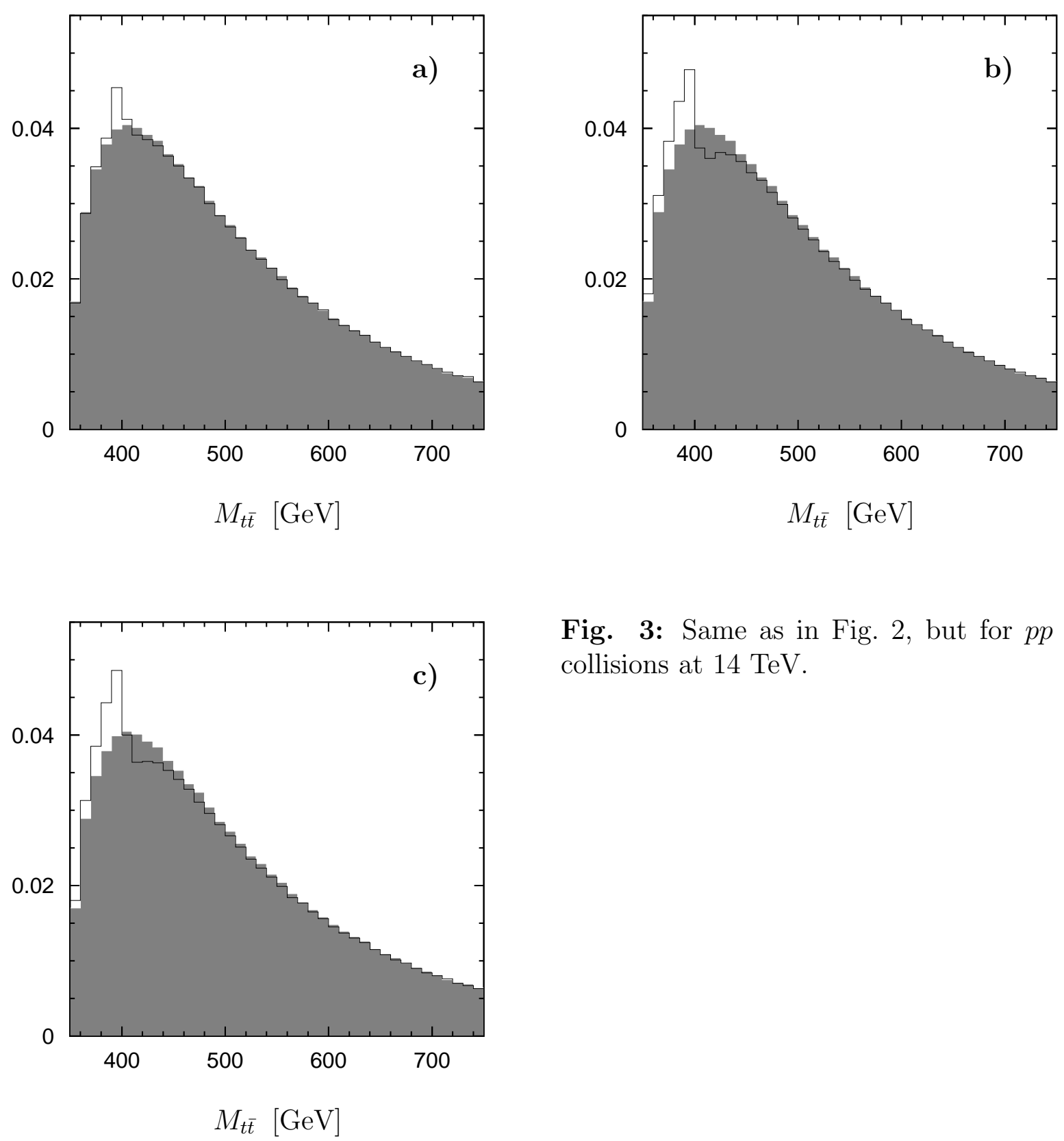

Fig. 3: Same as in Fig. 2, but for $p p$ collisions at $14 \mathrm{TeV}$. 
Figure 4
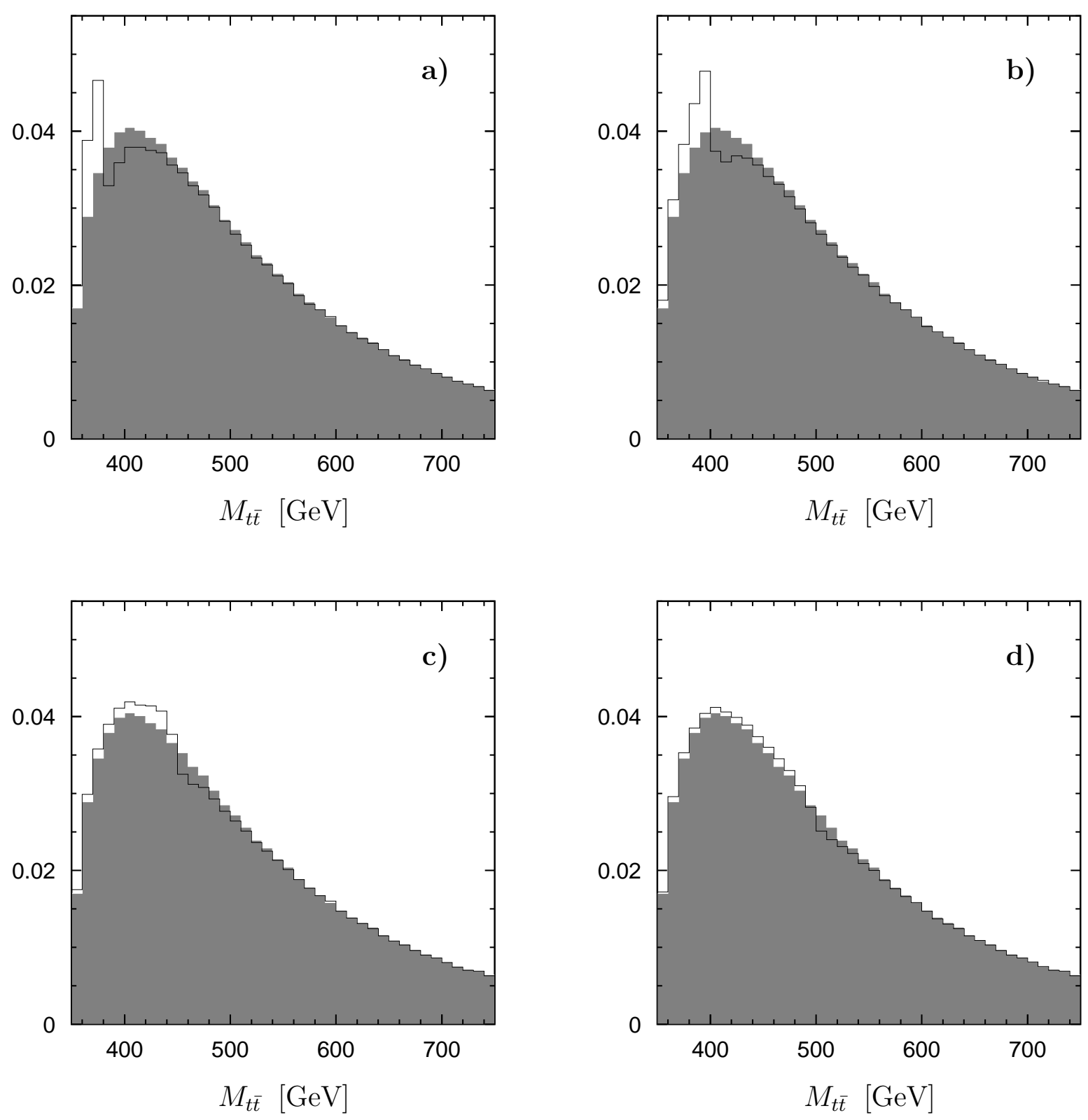

Fig. 4: Normalized $t \bar{t}$ invariant mass spectrum for $p p$ collisions at $14 \mathrm{TeV}$. Shown is the background contribution (grey) and the effects of a pseudoscalar Higgs boson with couplings $a=0, \tilde{a}=1$. The different plots a), b), c), d) are for mass values $m_{\varphi}=375,400,450,500 \mathrm{GeV}$, respectively. 
Figure 5

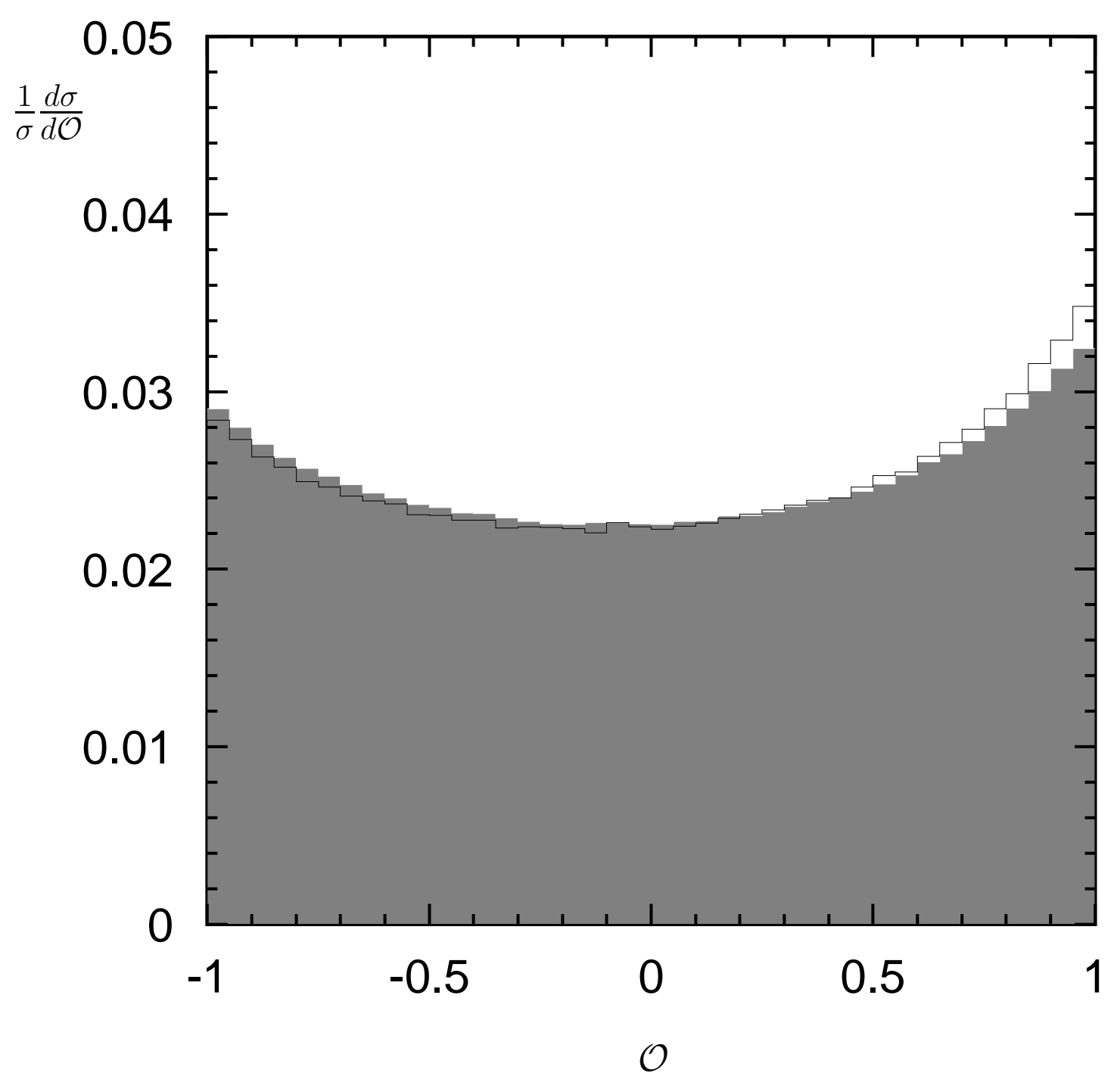

Fig. 5: Normalized distribution $\sigma^{-1} d \sigma / d \mathcal{O}$, where $\mathcal{O}$ is the cosine of the angle between the two leptons in the double lepton channel of $p p$ collisions at $\sqrt{s}=6 \mathrm{TeV}$. The grey area is the contribution from the background only. The solid line shows the effect of a pseudoscalar Higgs boson with mass $m_{\varphi}=400 \mathrm{GeV}$ and couplings $a=0, \tilde{a}=2$. 


\section{Figure 6}

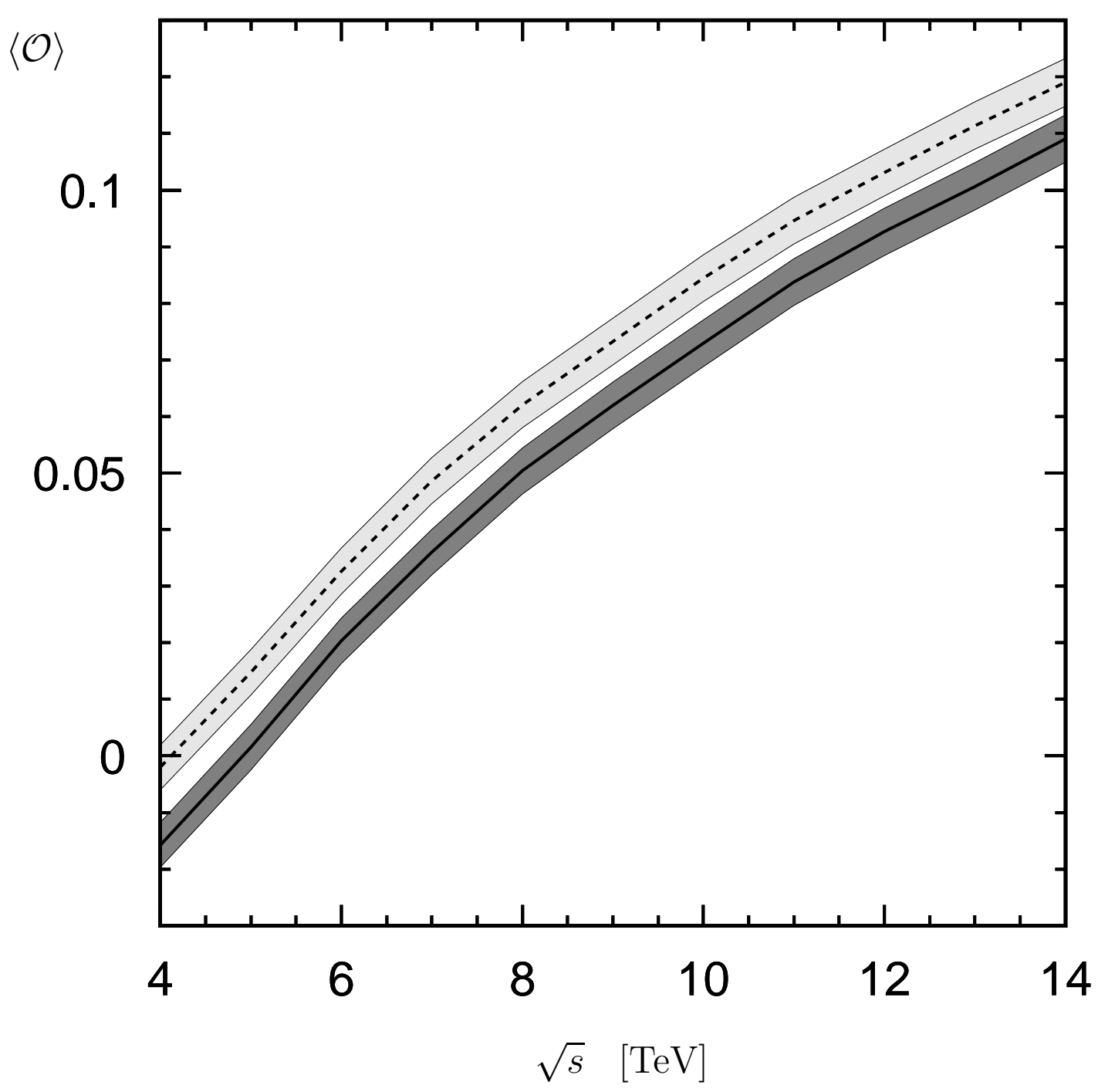

Fig. 6: Expectation value $\langle\mathcal{O}\rangle$ of the cosine of the angle spanned by the two leptons in the double lepton channel of $p p$ collisions, plotted as function of the c.m. energy $\sqrt{s}$. The solid line and the dark grey band represent the expectation from the background only, for $2 \times 10^{5}$ events at the $3 \sigma$ level. The dashed line with the light grey band was obtained with a pseudoscalar ( $a=0$, $\tilde{a}=2$ ) Higgs boson with mass $m_{\varphi}=400 \mathrm{GeV}$. 